\title{
Andersen Filtration and Hard Lefschetz
}

\author{
Wolfgang Soergel
}

August 10, 2021

For Joseph Bernstein

\begin{abstract}
On the space of homomorphisms from a Verma module to an indecomposable tilting module of the BGG-category $\mathcal{O}$ we define a natural filtration following Andersen And97 and establish a formula expressing the dimensions of the filtration steps in terms of coefficients of Kazhdan-Lusztig polynomials.
\end{abstract}

\section{Introduction}

Indecomposable tilting modules in category $\mathcal{O}$ were classified by Collingwood and Irving CI89 well before this terminology existed under the name of selfdual Verma flag modules. More precisely, they proved that applying the indecomposable projective functors of Bernstein-Gelfand to simple instead of projective Verma modules, you get precisely the indecomposable selfdual Verma flag modules instead of the indecomposable projectives, and that these indecomposable selfdual Verma flag modules nowadays called tilting modules are classified by their highest weight. Now we can define a filtration on the space of homomorphisms from a Verma module to a tilting module by analogy of what Andersen [And97] did in the algebraic group case. The main result of this article is a description of the dimensions of the subquotients of this filtration in terms of Kazhdan-Lusztig polynomials.

To be more precise, let me introduce some notation. Let $\mathfrak{g} \supset \mathfrak{b} \supset \mathfrak{h}$ be a semisimple complex Lie algebra, a Borel and a Cartan. Let $\rho \in \mathfrak{h}^{*}$ be the halfsum of roots from $\mathfrak{b}$ and let $\mathbb{C}[\mathbb{C} \rho]=T$ denote the regular functions on the line $\mathbb{C} \rho \subset \mathfrak{h}^{*}$. This is a quotient of $S \mathfrak{h}^{*}=\mathbb{C}[\mathfrak{h}]$, and every linear form $v: \mathbb{C} \rho \rightarrow \mathbb{C}$ defines an isomorphism with a polynomial ring $\mathbb{C}[v] \stackrel{\sim}{\rightarrow} T$. For a 
weight $\lambda \in \mathfrak{h}^{*}$ we form the Verma module $\Delta(\lambda)=U(\mathfrak{g}) \otimes_{U(\mathfrak{b})} \mathbb{C}_{\lambda} \in \mathfrak{g}$-mod and the deformed Verma module

$$
\Delta_{T}(\lambda)=U(\mathfrak{g}) \otimes_{U(\mathfrak{b})}\left(\mathbb{C}_{\lambda} \otimes T\right) \in \mathfrak{g}-\bmod -T
$$

Here and henceforth tensor products without any specification are to be understood over $\mathbb{C}$. The $T$-action is meant to only move the last tensor factor, however the $\mathfrak{b}$-action on $\mathbb{C}_{\lambda} \otimes T$ comes via the obvious surjection $\mathfrak{b} \rightarrow \mathfrak{h}$ from the $\mathfrak{h}$-action given by the tensor product action $H(a \otimes f)=\lambda(H) a \otimes f+a \otimes H f$ for $a \in \mathbb{C}_{\lambda}=\mathbb{C}$ and $f \in T$. Starting with the deformed Verma and taking the $T$-dual "weight space by weight space" and twisting the $\mathfrak{g}$-action on the result with a Chevalley automorphism we also get a deformed dual Verma module $\nabla_{T}(\lambda) \in \mathfrak{g}$-mod- $T$. The universal properties of Verma modules will then lead to a canonical embedding

$$
\operatorname{can}: \Delta_{T}(\lambda) \hookrightarrow \nabla_{T}(\lambda)
$$

which gives an isomorphism between the (analogues of the) highest weight spaces and is in fact a basis of the $T$-module $\operatorname{Hom}_{\mathfrak{g}-T}\left(\Delta_{T}(\lambda), \nabla_{T}(\lambda)\right)$. The Jantzen filtration can be understood as the filtration of our Verma $\Delta(\lambda)$ by the images of the $\operatorname{can}^{-1}\left(\nabla_{T}(\lambda) v^{i}\right)$ for $i=0,1,2, \ldots$ under the natural projection $\Delta_{T}(\lambda) \rightarrow \Delta(\lambda)$. Next let $\nu \in \mathfrak{h}^{*}$ be such that the Verma module $\Delta(\nu)$ is simple and let $E \in \mathfrak{g}$-mod be finite dimensional. Then $E \otimes \Delta(\nu)$ is tilting and we may consider the composition pairing

$\operatorname{Hom}\left(\Delta_{T}(\lambda), E \otimes \Delta_{T}(\nu)\right) \times \operatorname{Hom}\left(E \otimes \Delta_{T}(\nu), \nabla_{T}(\lambda)\right) \rightarrow \operatorname{Hom}\left(\Delta_{T}(\lambda), \nabla_{T}(\lambda)\right)$

where homomorphisms are understood in the category of $\mathfrak{g}$ - $T$-bimodules. As we remarked already, the pairing essentially lands in $T$. Furthermore we will prove that the paired spaces actually are free of finite rank over $T$, thus our pairing can be rewritten as a map, actually an embedding

$$
\operatorname{Hom}\left(\Delta_{T}(\lambda), E \otimes \Delta_{T}(\nu)\right) \hookrightarrow \operatorname{Hom}\left(E \otimes \Delta_{T}(\nu), \nabla_{T}(\lambda)\right)^{*}
$$

with the $*$ meaning a $T$-dual. Andersen's filtration is defined by taking on the right side of this embedding the filtration obtained by multiplying with the $v^{i}$ from the right, then taking the preimage of this filtration under our embedding, and finally the image of this preimage under the projection onto $\operatorname{Hom}_{\mathfrak{g}}(\Delta(\lambda), E \otimes \Delta(\nu))$ specializing $v$ to 0 alias applying $\otimes_{T} \mathbb{C}$.

In this paper we explain how to calculate the dimensions of the subquotients $\bar{F}^{i}$ of this Andersen filtration on $\operatorname{Hom}_{\mathfrak{g}}(\Delta(\lambda), E \otimes \Delta(\nu))$. More precisely, we identify the dimensions of the subquotients of the induced filtration on $\operatorname{Hom}_{\mathfrak{g}}(\Delta(\lambda), K)$ for $K \subset E \otimes \Delta(\nu)$ an indecomposable direct summand with 
coefficients of Kazhdan-Lusztig polynomials $P_{y, x}(q)$ as they are introduced in [KL79. And to be completely explicit, the general formula we prove as Theorem 4.4 means in the principal block for arbitrary $x, y$ in the Weyl group the formula

$$
\sum_{i \geq 0} \operatorname{dim}_{\mathbb{C}} \bar{F}^{i} \operatorname{Hom}_{\mathfrak{g}}(\Delta(-y \rho-\rho), K) q^{(l(x)-l(y)-i) / 2}=P_{y, x}(q)
$$

in case $K$ has highest weight $(-x \rho-\rho)$ and thus is the indecomposable tilting module $K=K(-x \rho-\rho)$ with this highest weight.

The proof given in the last section proceeds roughly speaking by translation to the Koszul-dual geometric side, where we run into the hard Lefschetz. More precisely, the embedding giving rise to Andersen's filtration is identified with the embedding of a costalk of the equivariant intersection cohomology complex of a Schubert variety into its stalk at the same point, both understood in the equivariant derived category of a point. This identification in turn passes through identifying both sides with the same construction in bimodules over polynomial rings, i.e. passing through a "coherent picture". More precisely, in sections 2-5 we explain the translation from category $\mathcal{O}$ to the coherent picture, culminating in 5.12 . The translation from geometry to the coherent picture is discussed thereafter.

The arguments given even show that the Andersen filtration coincides with the filtration on our spaces of homomorphisms coming from the $\mathbb{Z}$ graded structure introduced in [BGS96], although we do not make this explicit. This statement is very similar to the semisimplicity of the subquotients of the Jantzen filtration proved in BB93, but the method to obtain it is quite different. I would like to know how to directly relate both results, as this would give an alternative proof of the mentioned semisimplicity.

\section{Deformation of category $\mathcal{O}$}

2.1. In this and the next section we repeat results of [GJ81] in a language adapted to our goals, which is also very close to the language introduced in [Fie06. Let $S=S \mathfrak{h}=\mathbb{C}\left[\mathfrak{h}^{*}\right]$ be the symmetric algebra of $\mathfrak{h}$. We consider the category $\operatorname{Kring}^{S}$ of all commutative unitary rings $T$ with a distinguished morphism $\varphi: S \rightarrow T$. Given $T \in \operatorname{Kring}^{S}$ we consider the category $\mathfrak{g}-\operatorname{Mod}_{\mathbb{C}^{-}} T$ of all $\mathfrak{g}$ - $T$-bimodules on which the right and left actions of $\mathbb{C}$ coincide.

Definition 2.2. For $T=(T, \varphi) \in \operatorname{Kring}^{S}$ we define in any bimodule $M \in$ $\mathfrak{g}-\operatorname{Mod}_{\mathbb{C}^{-}} T$ for any $\lambda \in \mathfrak{h}^{*}$ the deformed weight space $M^{\lambda}$ by the formula

$$
M^{\lambda}=M_{T}^{\lambda}=\{m \in M \mid(H-\lambda(H)) m=m \varphi(H) \quad \forall H \in \mathfrak{h}\}
$$


2.3. For $M \in \mathfrak{g}-\operatorname{Mod}_{\mathbb{C}^{-}} T$ the canonical map from the direct sum of its deformed weight spaces to $M$ is always an injection $\bigoplus_{\lambda} M^{\lambda} \hookrightarrow M$. For $T=\mathbb{C}\left[\mathfrak{h}^{*}\right]$ this is evident, since the weight spaces $M^{\lambda}$ considered as $T \otimes T$ modules have support in the graphs of $(\lambda+): \mathfrak{h}^{*} \rightarrow \mathfrak{h}^{*}$ and these graphs are pairwise disjoint. In general our weight spaces have support in the preimage of our graphs under the map $\operatorname{Spec}\left(\mathbb{C}\left[\mathfrak{h}^{*}\right] \otimes T\right) \rightarrow \operatorname{Spec}\left(\mathbb{C}\left[\mathfrak{h}^{*}\right] \otimes \mathbb{C}\left[\mathfrak{h}^{*}\right]\right)$ induced by id $\otimes \varphi$ and thus are disjoint as well.

Definition 2.4. For every $T \in \mathrm{Kring}^{S}$ we define in our category of bimodules a full subcategory, the deformed category

$$
\mathcal{O}(T) \subset \mathfrak{g}-\operatorname{Mod}_{\mathbb{C}^{-}} T
$$

as the category of all bimodules $M$ which are locally finite for $\mathfrak{n}=[\mathfrak{b}, \mathfrak{b}]$ and decompose as the direct sum $M=\bigoplus M^{\lambda}$ of their deformed weight spaces.

2.5. Prominent objects of this category are the deformed Verma modules

$$
\Delta_{T}(\lambda)=\operatorname{prod}_{\mathfrak{b}} \mathfrak{g}\left(\mathbb{C}_{\lambda} \otimes T\right)=U(\mathfrak{g}) \otimes_{U(\mathfrak{b})}\left(\mathbb{C}_{\lambda} \otimes T\right)
$$

for $\lambda \in \mathfrak{h}^{*}$, where it is understood that the right action of $T$ acts only on the last tensor factor, whereas the left action comes from the left action of $U(\mathfrak{b})$ on $\mathbb{C}_{\lambda} \otimes T$ which we get via the canonical surjection $\mathfrak{b} \rightarrow \mathfrak{h}$ from the tensor action of $\mathfrak{h}$, where $H \in \mathfrak{h}$ acts on $\mathbb{C}_{\lambda}$ via the scalar $\lambda(H)$ and on $T$ by multiplication with $\varphi(H)$.

2.6. The category $\mathcal{O}(T)$ is stable under tensoring from the left with finite dimensional representations of $\mathfrak{g}$, where as left action of $\mathfrak{g}$ on such a tensor product we understand the tensor action and as right action of $T$ its right action on the second tensor factor. Along with a bimodule $\mathcal{O}(T)$ also contains all its subquotients. In case $T=\mathbb{C}$ and $\varphi$ the evaluation at the zero of $\mathfrak{h}^{*}$, the category $\mathcal{O}(T)$ specializes up to some missing finiteness conditions to the usual category $\mathcal{O}$ of Bernstein-Gelfand-Gelfand, and $\Delta_{\mathbb{C}}(\lambda)=\Delta(\lambda)$ is the Verma module with highest weight $\lambda$.

Definition 2.7. We now consider the opposed Borel of $\mathfrak{b}$ with respect to $\mathfrak{h}$ to be denoted $\overline{\mathfrak{b}} \subset \mathfrak{g}$ and for $\lambda \in \mathfrak{h}^{*}$ consider the subbimodule

$$
\nabla_{T}(\lambda) \subset \operatorname{ind}_{\overline{\mathfrak{b}}}^{\mathfrak{g}}\left(\mathbb{C}_{\lambda} \otimes T\right)=\operatorname{Hom}_{U(\overline{\mathfrak{b}})}\left(U(\mathfrak{g}), \mathbb{C}_{\lambda} \otimes T\right)
$$

defined as the sum of all deformed weight spaces of the Hom-space in question. We call it the deformed Nabla-module of highest weight $\lambda$. 
2.8. Under the identification given by restriction of our Hom-spaces with $\operatorname{Hom}_{\mathbb{C}}\left(U(\mathfrak{n}), \mathbb{C}_{\lambda} \otimes T\right)$ our $\nabla_{T}(\lambda)$ corresponds to those homomorphisms, which are different from zero on at most finitely many $\mathfrak{h}$-weight spaces of $U(\mathfrak{n})$. The deformed nablas also belong to $\mathcal{O}(T)$.

2.9. All weight spaces of $\nabla_{T}(\lambda)$ and $\Delta_{T}(\lambda)$ are free over $T$ and finitely generated, and if $T$ is not zero, the deformed weight spaces of weight $(\lambda-\nu)$ in both modules have the rank $\operatorname{dim}_{\mathbb{C}} U(\mathfrak{n})^{\nu}$. We have canonical morphisms $T \stackrel{\sim}{\rightarrow} \Delta_{T}(\lambda)^{\lambda} \hookrightarrow \Delta_{T}(\lambda)$ and $\nabla_{T}(\lambda) \rightarrow \nabla_{T}(\lambda)^{\lambda} \stackrel{\sim}{\rightarrow} T$ of $T$-modules and for any extension $T \rightarrow T^{\prime}$ canonical isomorphisms $\Delta_{T}(\lambda) \otimes_{T} T^{\prime} \stackrel{\sim}{\rightarrow} \Delta_{T^{\prime}}(\lambda)$ and $\nabla_{T}(\lambda) \otimes_{T} T^{\prime} \stackrel{\sim}{\rightarrow} \nabla_{T^{\prime}}(\lambda)$.

2.10. We now choose for our Lie algebra an involutive automorphism $\tau: \mathfrak{g} \rightarrow$ $\mathfrak{g}$ with $\left.\tau\right|_{\mathfrak{h}}=-$ id and define a contravariant functor

$$
d=d_{\tau}: \mathfrak{g}-\operatorname{Mod}_{\mathbb{C}^{-}} T \rightarrow \mathfrak{g}-\operatorname{Mod}_{\mathbb{C}^{-}} T
$$

by letting $d M \subset \operatorname{Hom}_{-T}(M, T)^{\tau}$ be the sum of all deformed weight spaces in the space of homomorphisms of right $T$-modules from $M$ to $T$ with its contragredient $\mathfrak{g}$-action twisted by $\tau$. If $M \in \mathfrak{g}-\operatorname{Mod}_{\mathbb{C}^{-}} T$ is the sum of its deformed weight spaces, we have a canonical morphism $M \rightarrow d d M$, and if in addition all deformed weight spaces of $M$ are free and finitely generated over $T$, this canonical morphism is an isomorphism.

2.11. The restriction onto the highest deformed weight space defines together with the universal property of the induced representation a canonical homomorphism

$$
\operatorname{Hom}_{-T}\left(\operatorname{prod}_{\overline{\mathfrak{b}}}^{\mathfrak{g}}\left(\mathbb{C}_{-\lambda} \otimes T\right), T\right) \rightarrow \operatorname{ind}_{\mathfrak{b}}^{\mathfrak{g}} \operatorname{Hom}_{-T}\left(\mathbb{C}_{\lambda} \otimes T, T\right)
$$

and considering the deformed weight spaces we see that it induces an isomorphism of bimodules

$$
d \Delta_{T}(\lambda) \stackrel{\sim}{\rightarrow} \nabla_{T}(\lambda) .
$$

With our preceding remarks we also get $d \nabla_{T}(\lambda) \cong \Delta_{T}(\lambda)$. By the tensor identity, i.e. since tensoring with a representation of a Lie algebra commutes with tensor-inducing a representation from a subalgebra, furthermore $E \otimes$ $\Delta_{T}(\lambda)$ admits a filtration with subquotients $\Delta_{T}(\lambda+\nu)$, where $\nu$ runs over the multiset $P(E)$ of weights of $E$. Since $E \otimes$ ? commutes up to the choice of an isomorphism $d E \cong E$ with our duality $d$, we deduce an analogous result for $E \otimes \nabla_{T}(\lambda)$.

Proposition 2.12. 1. For all $\lambda$ the restriction to the deformed weight space of $\lambda$ together with the two canonical identifications $\Delta_{T}(\lambda)^{\lambda} \stackrel{\sim}{\rightarrow} T$ and $\nabla_{T}(\lambda)^{\lambda} \stackrel{\sim}{\rightarrow} T$ induces an isomorphism

$$
\operatorname{Hom}_{\mathcal{O}(T)}\left(\Delta_{T}(\lambda), \nabla_{T}(\lambda)\right) \stackrel{\sim}{\rightarrow} T
$$


2. For $\lambda \neq \mu$ in $\mathfrak{h}^{*}$ we have $\operatorname{Hom}_{\mathcal{O}(T)}\left(\Delta_{T}(\lambda), \nabla_{T}(\mu)\right)=0$.

3. For all $\lambda, \mu \in \mathfrak{h}^{*}$ we have $\operatorname{Ext}_{\mathcal{O}(T)}^{1}\left(\Delta_{T}(\lambda), \nabla_{T}(\mu)\right)=0$.

Proof. We prove (3), the simpler case of spaces of homomorphisms is treated in the same way. Let $R^{+} \subset \mathfrak{h}^{*}$ denote the roots of $\mathfrak{n}$ and $\left|R^{+}\right\rangle \subset \mathfrak{h}^{*}$ the submonoid generated by $R^{+}$and $\leq$the partial order on $\mathfrak{h}^{*}$ with $\lambda \leq \mu \Leftrightarrow$ $\mu \in \lambda+\left|R^{+}\right\rangle$. Every short exact sequence $\nabla_{T}(\mu) \hookrightarrow M \rightarrow \Delta_{T}(\lambda)$ with $M \in \mathcal{O}(T)$ and $\lambda \not \leq \mu$ splits, since any preimage in $M^{\lambda}$ of the canonical generator of $\Delta_{T}(\lambda)$ already is annihilated by $\mathfrak{n}$ and thus induces a splitting. In case $\lambda \leq \mu$ we use our duality $d$ to pass to the dual situation. This proves the triviality of the extension in question.

Corollary 2.13. Let $M, N \in \mathcal{O}(T)$. If $M$ is a direct summand of an object with finite $\Delta_{T}$-flag and $N$ a direct summand of an object with finite $\nabla_{T^{-}}$ flag, then the space of homomorphism $\operatorname{Hom}_{\mathcal{O}(T)}(M, N)$ is a finitely generated projective $T$-module and for any ring extension $T \rightarrow T^{\prime}$ the obvious map defines an isomorphism

$$
\operatorname{Hom}_{\mathcal{O}(T)}(M, N) \otimes_{T} T^{\prime} \stackrel{\sim}{\rightarrow} \operatorname{Hom}_{\mathcal{O}\left(T^{\prime}\right)}\left(M \otimes_{T} T^{\prime}, N \otimes_{T} T^{\prime}\right)
$$

Proof. This follows directly from 2.12 by induction on the lengths of the flags.

2.14. If $Q \in \mathrm{Kring}^{S}$ is a field and if for all roots $\alpha$ the coroots $\alpha^{\vee}$ are not mapped to $\mathbb{Z} \subset Q$ under $S \rightarrow Q$, then the category $\mathcal{O}(Q)$ is semisimple, (i.e. all surjections split) and its simple objects are the $\Delta_{Q}(\lambda)=\nabla_{Q}(\lambda)$ for $\lambda \in \mathfrak{h}^{*}$.

\section{Deforming indecomposable tilting modules}

3.1. Let $D=S_{(0)}$ be the local ring at zero of $\mathfrak{h}^{*}$. For $\lambda \in \mathfrak{h}^{*}$ with $\Delta(\lambda)$ simple the canonical map defines an isomorphism

$$
\Delta_{D}(\lambda) \stackrel{\sim}{\rightarrow} \nabla_{D}(\lambda)
$$

Indeed, we only need to show that this map gives isomorphisms on all deformed weight spaces, and these are free of finite rank over the local ring $D$. By Nakayama's Lemma we thus only need to show that our map becomes an isomorphism under ? $\otimes_{D} \mathbb{C}$, and this follows directly from the simplicity assumption on $\Delta(\lambda)$.

Definition 3.2. Given $T \in \operatorname{Kring}^{S}$ let $\mathcal{K}(T) \subset \mathcal{O}(T)$ denote the smallest subcategory, which 
1. contains all $\Delta_{T}(\lambda)$ for which the canonical map gives an isomorphism $\Delta_{T}(\lambda) \stackrel{\sim}{\rightarrow} \nabla_{T}(\lambda)$,

2. is stable under tensoring with finite dimensional representations of $\mathfrak{g}$,

3. is stable under forming direct summands.

We call $\mathcal{K}(T)$ the category of $T$-deformed tilting modules.

3.3. For $\mathbb{C}=\mathbb{C}_{0} \in \mathrm{Kring}^{S}$ the objects of $\mathcal{K}(\mathbb{C})$ are the tilting modules of the usual BGG-category $\mathcal{O}$.

Proposition 3.4. If $T \in \mathrm{Kring}^{S}$ is a complete local ring "under $S$ " such that the preimage in $S$ of its maximal ideal is just the vanishing ideal of the origin in $\mathfrak{h}^{*}$, then the specialization

$$
? \otimes_{T} \mathbb{C}: \mathcal{K}(T) \rightarrow \mathcal{K}(\mathbb{C})
$$

induces a bijection on isomorphism classes, and under this bijection indecomposables correspond to indecomposables.

Proof. The tilting modules from $\mathcal{O}$ are precisely the direct summands of tensor products of simple Vermas with finite dimensional representations. All such tensor products $K \in \mathcal{K}(\mathbb{C})$ lift by definition. If $K_{T} \in \mathcal{K}(T)$ is such a lift, we deduce from 2.13 that the canonical map leads to an isomorphism $\mathbb{C} \otimes_{T}$ End $K_{T} \stackrel{\sim}{\rightarrow}$ End $K_{\mathbb{C}}$ of finite dimensional $\mathbb{C}$-algebras. General results [Ben91 or [CR90], I, (6.7) concerning the lifting of idempotents now show that any projection of $K_{\mathbb{C}}$ to a direct summand can be lifted to a projection of $K_{T}$ to a direct summand, which gives surjectivity on isomorphism classes in our Proposition. The same argument, now applied to an arbitrary $K \in \mathcal{K}(\mathbb{C})$, shows that only indecomposable objects from $\mathcal{K}(T)$ can go to indecomposable objects from $\mathcal{K}(\mathbb{C})$. Similarily, any lifting of an isomorphism has to be an isomorphism, since every lift of a unit in an endomorphism ring has to be a unit, and this establishes the claimed bijection on isomorphism classes.

3.5. For $\lambda \in \mathfrak{h}^{*}$ we let $K_{T}(\lambda) \in \mathcal{K}(T)$ denote the $T$-deformation of the indecomposable tilting module $K(\lambda) \in \mathcal{O}$ with highest weight $\lambda$.

\section{The Andersen filtration}

4.1. Fix $K \in \mathfrak{g}-\operatorname{Mod}_{\mathbb{C}^{-}} T$ and $\lambda \in \mathfrak{h}^{*}$. To increase readability we use the abbreviations $\Delta_{T}(\lambda)=\Delta, \nabla_{T}(\lambda)=\nabla$ and $\operatorname{Hom}_{\mathfrak{g}-T}=$ Hom and consider the $T$-bilinear pairing

$$
\operatorname{Hom}(\Delta, K) \times \operatorname{Hom}(K, \nabla) \rightarrow \operatorname{Hom}(\Delta, \nabla)=T
$$


given by composition. If for any $T$-module $H$ we denote by $H^{*}$ the $T$-module $\operatorname{Hom}_{T}(M, T)$, then our pairing induces a map

$$
E=E_{\lambda}(K): \operatorname{Hom}(\Delta, K) \rightarrow \operatorname{Hom}(K, \nabla)^{*}
$$

If $K$ is tilting, then by 2.13 our map $E$ is a map between finitely generated projective $T$-modules. If in addition $T \in \mathrm{Kring}^{S}$ is an integral domain and $Q=$ Quot $T$ satisfies the assumptions of remark 2.14, thus $\mathcal{O}(Q)$ is semisimple with simple objects $\Delta_{Q}(\lambda)=\nabla_{Q}(\lambda)$, then our pairing is nondegenerate over $Q$ and our map $E_{\lambda}(K)$ induces an isomorphism over $Q$ and in particular is an injection. If now $T=\mathbb{C}[[v]]$ is the ring of formal power series around the origin on a line $\mathbb{C} \delta \subset \mathfrak{h}^{*}$, which isn't contained in any reflection hyperplane of the Weyl group, then $Q=$ Quot $\mathbb{C}[[v]]$ satisfies our assumptions of remark 2.14. If now $K \in \mathcal{K}(\mathbb{C}[[v]])$ is a deformed tilting module, we can use the embedding

$$
E_{\lambda}(K): \operatorname{Hom}(\Delta, K) \hookrightarrow \operatorname{Hom}(K, \nabla)^{*}
$$

of free $\mathbb{C}[[v]]$-modules of finite rank to restrict the obvious filtration of the right hand side by the $v^{i} \operatorname{Hom}(K, \nabla)^{*}$ and thus get a filtration on $\operatorname{Hom}(\Delta, K)=$ $\operatorname{Hom}_{\mathfrak{g}-\mathbb{C}[[v]]}\left(\Delta_{\mathbb{C}[[v]]}(\lambda), K\right)$.

Definition 4.2. Given $K_{\mathbb{C}} \in \mathcal{K}(\mathbb{C})$ a tilting module of $\mathcal{O}$ and $K \in \mathcal{K}(\mathbb{C}[[v]])$ a $\mathbb{C}[[v]]$-deformation of $K_{\mathbb{C}}$ in the sense of 3.4 with $S \rightarrow \mathbb{C}[[v]]$ the restriction to a formal neighbourhood of the origin in the line $\mathbb{C} \rho$ with $\rho$ as in 5.3 , the image of the filtration defined above under specialization ? $\otimes_{\mathbb{C}[[v]]} \mathbb{C}$ will be called the Andersen-filtration on $\operatorname{Hom}_{\mathfrak{g}}\left(\Delta(\lambda), K_{\mathbb{C}}\right)$.

4.3. We leave it to the reader to show that this filtration is independent of the choice of the deformation, which is only well defined up to isomorphism. The goal of this work is to determine the dimensions of the subquotients of the Andersen filtration on $\operatorname{Hom}_{\mathfrak{g}}(\Delta(\lambda), K(\mu))$ for all $\lambda, \mu \in \mathfrak{h}^{*}$ or more precisely their description as coefficients of Kazhdan-Lusztig polynomials.

Theorem 4.4. The dimensions of the subquotients of the Andersen filtrations satisfy the identities

$$
\sum_{i} \operatorname{dim}_{\mathbb{C}} \bar{F}^{i} \operatorname{Hom}_{\mathfrak{g}}\left(\Delta\left(\lambda_{\bar{y}}\right), K\left(\lambda_{\bar{x}}\right)\right) q^{(l(x)-l(y)-i) / 2}=P_{y, x}(q)
$$

4.5. The proof will be given only in the last section, but let me explain here what all this notation means. We start with a $\rho$-dominant weight $\lambda \in \mathfrak{h}_{\text {dom }}^{*}$ in the sense of 5.3. It gives two subgroups $W_{\bar{\lambda}} \supset W_{\lambda}$ of the Weyl group as explained in 5.6, and $\bar{x}, \bar{y}$ denote cosets of $W_{\bar{\lambda}} / W_{\lambda}$ with $x, y$ their longest 
representatives. Finally $\lambda_{\bar{x}}=w_{\bar{\lambda}} \bar{x} \cdot \lambda$ is to be understood as in 10.2 with $w_{\bar{\lambda}}$ the longest element of $W_{\bar{\lambda}}$, and $P_{y, x}$ is the Kazhdan-Lusztig polynomial with respect to the Coxeter group $W_{\bar{\lambda}}$ and its length function $l$. In fact the arguments given in this article show that the Andersen filtration coincides with the grading filtration induced from the graded version of $\mathcal{O}$, but I felt incapable to explain this in the framework of this article.

4.6. The Jantzen filtration on a Verma module $\Delta(\lambda)$ certainly induces a filtration on $\operatorname{Hom}_{\mathfrak{g}}(P(\mu), \Delta(\lambda))$ for $P(\mu) \rightarrow \Delta(\mu)$ the indecomposable projective cover of $\Delta(\mu)$ in $\mathcal{O}$. This filtration in turn comes in the same way from the embedding $\Delta_{\mathbb{C}[[v]]}(\lambda) \rightarrow \nabla_{\mathbb{C}[v]]}(\lambda)$ or more precisely the embeddings

$$
\operatorname{Hom}_{\mathfrak{g}-\mathbb{C}[[v]]}\left(P_{\mathbb{C}[[v]]}(\mu), \Delta_{\mathbb{C}[[v]]}(\lambda)\right) \hookrightarrow \operatorname{Hom}_{\mathfrak{g}-\mathbb{C}[[v]]}\left(P_{\mathbb{C}[[v]]}(\mu), \nabla_{\mathbb{C}[[v]]}(\lambda)\right)
$$

induced by them, where $P_{\mathbb{C}[[v]]}(\mu) \rightarrow \Delta_{\mathbb{C}[[v]]}(\mu)$ are the indecomposable projective covers in $\mathcal{O}(\mathbb{C}[[v]])$. This shows the analogy of both filtrations. In fact, the contravariant equivalence explained in Soe97a from the category of Verma flag modules to itself, mapping projectives to tilting modules, induces a map on homomorphism spaces, and this map should identify both filtrations. However I cannot prove this without using the Jantzen conjecture.

\section{Deformed translation}

5.1. Let $Z \subset U(\mathfrak{g})$ be the center, so that $Z \otimes T$ acts on any bimodule $M \in \mathfrak{g}-\operatorname{Mod}_{\mathbb{C}^{-}} T$. We now consider the push-out diagram of $\mathbb{C}$-algebras

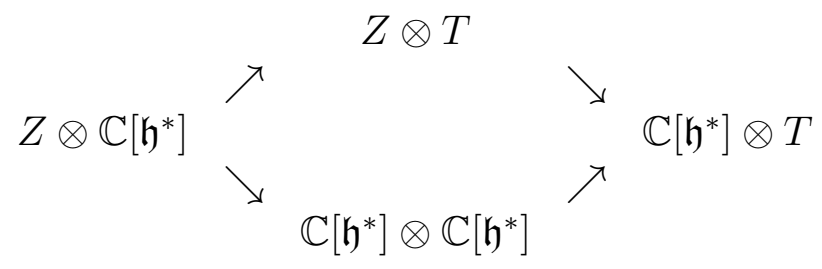

where for $\xi: Z \rightarrow \mathbb{C}\left[\mathfrak{h}^{*}\right]$ we always take the variant of the Harish-Chandra homomorphism with $\xi(z)-z \in U \mathfrak{n}$. It leads to a finite ring extension and the same holds thus also for both downward arrows of our diagram. The graph of the addition of $\lambda \in \mathfrak{h}^{*}$ is an irreducible closed subset of $\operatorname{Spec}\left(\mathbb{C}\left[\mathfrak{h}^{*}\right] \otimes \mathbb{C}\left[\mathfrak{h}^{*}\right]\right)$ and the same holds for its image in $\operatorname{Spec}\left(Z \otimes \mathbb{C}\left[\mathfrak{h}^{*}\right]\right)$. The preimage in $\operatorname{Spec}(Z \otimes T)$ of this image will be denoted $\Xi_{\lambda} \subset \operatorname{Spec}(Z \otimes T)$. By definition $\Delta_{T}(\lambda)$ and $\nabla_{T}(\lambda)$ both have support in $\Xi_{\lambda}$ as $Z \otimes T$-modules.

Lemma 5.2. The support in $\operatorname{Spec}(Z \otimes T)$ of any element of an object $M \in$ $\mathcal{O}(T)$ is contained in a finite union of sets of the form $\Xi_{\lambda}$ with $\lambda \in \mathfrak{h}^{*}$. 
Proof. Let $v$ be our element. We may assume $v \in M^{\lambda}$ for some $\lambda \in \mathfrak{h}^{*}$. We may further assume the submodule generated by $v$ to be contained in $\bigoplus_{\mu \leq \nu} M^{\lambda+\mu}$ for any integral dominant weight $\nu \in X^{+}$. The object

$$
U(\mathfrak{g}) \otimes_{U(\mathfrak{b})} \tau_{\leq \lambda+\nu}\left(U(\mathfrak{b}) \otimes_{U(\mathfrak{h})}\left(\mathbb{C}_{\lambda} \otimes T\right)\right)
$$

with hopefully selfexplaining $\tau_{\leq \lambda+\nu}$ has a finite $\Delta_{T}$-flag and our $v$ is contained in the image of a homomorphism of said object to $M$.

Definition 5.3. Let $\rho=\rho\left(R^{+}\right)$be the halfsum of positive roots. We put

$$
\mathfrak{h}_{\text {dom }}^{*}=\left\{\lambda \in \mathfrak{h}^{*} \mid\left\langle\lambda+\rho, \alpha^{\vee}\right\rangle \notin\{-1,-2, \ldots\} \forall \alpha \in R^{+}\right\}
$$

and call the elements of this set $\rho$-dominant weights. We use the usual notation $w \cdot \lambda=w(\lambda+\rho)-\rho$ for the action of the Weyl group translated to the fixed point $-\rho$.

Theorem 5.4 (Decomposition of deformed categories). Let $T$ be an $S_{(0)}$ ring, i.e. the morphism $S \rightarrow T$ should factor through the local ring $S_{(0)}$ of $\mathfrak{h}^{*}$ at the origin. Then we have a decomposition

$$
\mathcal{O}(T)=\prod_{\lambda \in \mathfrak{h}_{\mathrm{dom}}^{*}} \mathcal{O}_{\lambda}(T)
$$

where $\mathcal{O}_{\lambda}(T)$ consists of all $M \in \mathcal{O}(T)$ which satisfy $M=\bigoplus_{\nu \in \lambda+\mathbb{Z} R} M^{\nu}$ and $\operatorname{supp}_{Z \otimes T} M \subset \bigcup_{w \in W} \Xi_{w \cdot \lambda}$.

Proof. From $\Xi_{\lambda} \cap \Xi_{\mu} \neq \emptyset$ we get for $T=S_{(0)}$ already $W \cdot \lambda=W \cdot \mu$. The rest of the argument can be copied from the case $T=\mathbb{C}$, see [BG80].

5.5. As in the non-deformed case we have for $\lambda, \mu \in \mathfrak{h}_{\text {dom }}^{*}$ with integral difference $\lambda-\mu \in X$ translation functors

$$
T_{\lambda}^{\mu}: \mathcal{O}_{\lambda}(T) \rightarrow \mathcal{O}_{\mu}(T)
$$

which are exact, satisfy adjunctions $\left(T_{\lambda}^{\mu}, T_{\mu}^{\lambda}\right)$ and have all the usual properties. We call them deformed translations. The category of deformed tilting modules in one of our blocks will be denoted $\mathcal{K}(T) \cap \mathcal{O}_{\lambda}(T)=\mathcal{K}_{\lambda}(T)$.

5.6. Let us put $D=S_{(0)}$. If $T$ is a $D$-algebra, then for $\lambda \in \mathfrak{h}_{\text {dom }}^{*}$ the deformed Verma module $\Delta_{T}(\lambda)$ is projective in $\mathcal{O}_{\lambda}(T)$. The isotropy group of a weight $\lambda \in \mathfrak{h}^{*}$ under the dot-action of the Weyl group will be denoted $W_{\lambda}$, the isotropy group of its coset $\bar{\lambda}=\lambda+\langle R\rangle$ under the root lattice will be denoted $W_{\bar{\lambda}}$. The longest element of $W_{\bar{\lambda}}$ will be denoted $w_{\bar{\lambda}}$, the rings of invariants for the natural actions of $W_{\lambda} \subset W_{\bar{\lambda}}$ on $D$ will be denoted $D^{\lambda} \supset D^{\bar{\lambda}}$. 
Theorem 5.7 (Deformation of projectives). The functor ? $\otimes_{D} \mathbb{C}: \mathcal{O}(D) \rightarrow$ $\mathcal{O}(\mathbb{C})$ induces a bijection between isomorphism classes of finitely generated projective objects in both categories.

Proof. [Soe90].

Definition 5.8. Given $\lambda \in \mathfrak{h}^{*}$ let $P_{D}(\lambda) \in \mathcal{O}(D)$ denote the finitely generated projective specalizing to $P(\lambda)$ under ? $\otimes_{D} \mathbb{C}$. We call it the deformation of the projective $P(\lambda)$. Given $\lambda \in \mathfrak{h}_{\text {dom }}^{*}$ we use for the deformed antidominant projective the abbreviation $P_{D}\left(w_{\bar{\lambda}} \cdot \lambda\right)=A_{D}(\lambda)=A(\lambda)$.

Theorem 5.9 (Endomorphisms of antidominant projectives). Given $\lambda \in$ $\mathfrak{h}_{\text {dom }}^{*}$ the multiplication defines a surjection $Z \otimes D \rightarrow \operatorname{End}_{\mathcal{O}(D)} A(\lambda)$. If $(+\lambda)^{\sharp}$ : $S \rightarrow S$ denotes the comorphism of $(+\lambda): \mathfrak{h}^{*} \rightarrow \mathfrak{h}^{*}$, then the composition

$$
Z \otimes D \stackrel{\xi \otimes \text { id }}{\longrightarrow} S \otimes D \stackrel{(+\lambda)^{\sharp} \otimes \text { id }}{\longrightarrow} S \otimes D \rightarrow D \otimes_{D^{\bar{\lambda}}} D
$$

has image $D^{\lambda} \otimes_{D^{\bar{\lambda}}} D$ and the same kernel as the surjection considered before and we thus get an isomorphism

$$
D^{\lambda} \otimes_{D^{\bar{\lambda}}} D \stackrel{\sim}{\rightarrow} \operatorname{End}_{\mathcal{O}(D)} A(\lambda)
$$

Proof. For $\lambda$ integral the proof is given in [Soe92]. The proof in general is essentially the same.

5.10. For better transparency we use hereafter frequently the notation $\operatorname{Hom}_{\mathcal{O}(D)}=$ Hom and $\operatorname{End}_{\mathcal{O}(D)}=$ End. Any choice of a deformed antidominant projective $A(\lambda)$ for $\lambda \in \mathfrak{h}_{\text {dom }}^{*}$ gives us via the rule $\mathbb{V}=\mathbb{V}_{D}=\operatorname{Hom}_{\mathcal{O}(D)}(A(\lambda)$, $)$ an exact functor

$$
\mathbb{V}: \mathcal{O}(D) \rightarrow D^{\lambda}-\operatorname{Mod}_{\mathbb{C}^{-}} D
$$

which is different from zero only on $\mathcal{O}_{\lambda}(D)$. If further $\mu \in \mathfrak{h}_{\text {dom }}^{*}$ is given with $\lambda-\mu \in X$ and $W_{\mu} \supset W_{\lambda}$ and if we choose an isomorphism $T_{\mu}^{\lambda} A(\mu) \stackrel{\sim}{\rightarrow} A(\lambda)$, we get a commutative diagram

$$
\begin{array}{cccc}
D^{\mu} \otimes_{D^{\bar{\mu}}} D & \stackrel{\sim}{\rightarrow} & \text { End } A(\mu) \\
\downarrow & & \downarrow \\
D^{\lambda} \otimes_{D^{\bar{\mu}}} D & \stackrel{\sim}{\rightarrow} & \text { End } A(\lambda)
\end{array}
$$

with the left vertical induced from the embedding $D^{\mu} \subset D^{\lambda}$ and the right vertical given by $T_{\mu}^{\lambda}$ and our isomorphism, see Soe92. If we fix such an isomorphism and in addition choose an adjunction $\left(T_{\mu}^{\lambda}, T_{\lambda}^{\mu}\right)$, then we get isomorphisms

$$
\operatorname{Hom}\left(A(\mu), T_{\lambda}^{\mu} M\right) \stackrel{\sim}{\rightarrow} \operatorname{Hom}\left(T_{\mu}^{\lambda} A(\mu), M\right) \stackrel{\sim}{\rightarrow} \operatorname{Hom}(A(\lambda), M)
$$


which lead to an isomorphism of functors, up to which the diagram

$$
\begin{array}{ccc}
\mathcal{O}_{\lambda}(D) & \stackrel{\mathbb{V}}{\rightarrow} & D^{\lambda}-\operatorname{Mod}_{\mathbb{C}^{-}} D \\
T_{\lambda}^{\mu} \downarrow & & \downarrow \text { res } \\
\mathcal{O}_{\mu}(D) & \stackrel{\mathbb{V}}{\rightarrow} & D^{\mu}-\operatorname{Mod}_{\mathbb{C}^{-}} D
\end{array}
$$

commutes. Using the adjunctions we also find an isomorphism of functors, up to which the diagram

$$
\begin{array}{ccc}
\mathcal{O}_{\mu}(D) & \stackrel{\mathbb{V}}{\rightarrow} & D^{\mu}-\operatorname{Mod}_{\mathbb{C}^{-}} D \\
T_{\mu}^{\lambda} \downarrow & & \downarrow D^{\lambda} \otimes_{D^{\mu}} ? \\
\mathcal{O}_{\lambda}(D) & \stackrel{\mathbb{V}}{\rightarrow} & D^{\lambda}-\operatorname{Mod}_{\mathbb{C}^{-}} D
\end{array}
$$

commutes.

5.11. Given $\lambda, \mu \in \mathfrak{h}_{\text {dom }}^{*}$ with integral difference one may more generally consider the translations $T_{\lambda}^{\mu}: \mathcal{O}_{\lambda}(D) \rightarrow \mathcal{O}_{\mu}(D)$ which can be written as $T_{\lambda}^{\mu} \cong T_{\nu}^{\mu} T_{\lambda}^{\nu}$ for one and any $\nu \in \mathfrak{h}_{\text {dom }}^{*}$ with integral difference to $\lambda$ and $\mu$ and the property $W_{\nu}=W_{\lambda} \cap W_{\mu}$. This can be deduced from the known effects on Verma modules using the classification of projective functors [BG80. If we form $D_{\lambda}^{\mu}=D^{W_{\lambda} \cap W_{\mu}} \in D^{\mu}-\operatorname{Mod}_{\mathbb{C}^{-}} D^{\lambda}$, then we may interpret both diagrams as one diagram commuting up to natural isomorphism, namely the diagram

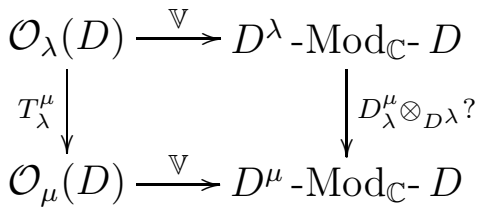

If we pass to the adjoints of the vertical functors, we get another diagram commuting up to natural isomorphism, namely

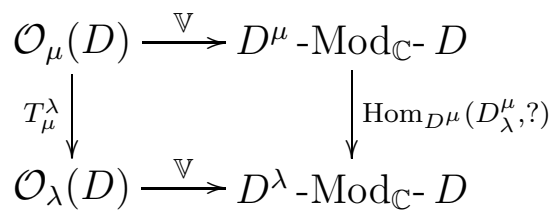

Theorem 5.12 (Struktursatz for deformed tilting modules). The functors $\mathbb{V}$ are fully faithful on deformed tilting modules. More precisely we have for any $\lambda \in \mathfrak{h}_{\mathrm{dom}}^{*}$ :

1. Given $K \in \mathcal{K}_{\lambda}(D)$ and $F \in \mathcal{O}_{\lambda}(D)$ an object with $\Delta_{D}$-flag the functor $\mathbb{V}$ induces an isomorphism

$$
\operatorname{Hom}_{\mathfrak{g}-D}(F, K) \stackrel{\sim}{\rightarrow} \operatorname{Hom}_{D^{\lambda}-D}(\mathbb{V} F, \mathbb{V} K)
$$


2. Given $K \in \mathcal{K}_{\lambda}(D)$ and $F \in \mathcal{O}_{\lambda}(D)$ an object with a $\nabla_{D}$-flag the functor $\mathbb{V}$ induces an isomorphism

$$
\operatorname{Hom}_{\mathfrak{g}-D}(K, F) \stackrel{\sim}{\rightarrow} \operatorname{Hom}_{D^{\lambda}-D}(\mathbb{V} K, \mathbb{V} F)
$$

5.13. In greater generality the first statement is proven as Theorem 10 in [Fie06]: The functors $\mathbb{V}$ are even fully faithful on arbitrary objects with a finite $\Delta_{D}$-flag.

5.14. In the non-deformed case $T=\mathbb{C}$ the functor $\mathbb{V}$ is fully faithful on the category of tilting modules of a given block. Indeed for any maximal ideal $\chi \subset Z$ and arbitrary projective functors $F, G: U / \chi U-\bmod \rightarrow U-\bmod$ and an arbitrary Verma module $\Delta$ with $\chi \Delta=0$ applying our functors to $\Delta$ defines a bijection

$$
\operatorname{Trans}_{U / \chi U}-\bmod \rightarrow(F, G) \stackrel{\sim}{\rightarrow} \operatorname{Hom}_{\mathfrak{g}}(F \Delta, G \Delta)
$$

where on the left hand side we mean the set of transformations from the functor $F$ to the functor $G$ and did only specify the start category, since this is the most subtle point in this business. For projective Vermas this is shown in [BG80], and since by [BGG75 the enveloping algebra surjects onto the ad-finite endomorphisms of every Verma, the proof given there works more generally for every Verma. The embedding of a simple Verma $\Delta_{e}$ into a projective Verma $\Delta_{p}$ thus gives bijections

$$
\operatorname{Hom}_{\mathfrak{g}}\left(F \Delta_{e}, G \Delta_{e}\right) \stackrel{\sim}{\rightarrow} \operatorname{Hom}_{\mathfrak{g}}\left(F \Delta_{p}, G \Delta_{p}\right)
$$

Since it also gives bijections $\mathbb{V} F \Delta_{e} \stackrel{\sim}{\rightarrow} \mathbb{V} F \Delta_{p}$, the claim follows. In the nondeformed case however the faithfulness on morphisms from tiltings to dual Vermas or from Vermas to tiltings does not hold.

Proof. Given $\lambda, \mu \in \mathfrak{h}_{\text {dom }}^{*}$ with integral difference let $D_{\mu}^{\lambda} \in D^{\lambda}-\operatorname{Mod}_{\mathbb{C}^{-}} D^{\mu}$ be the bimodule $D^{W_{\lambda} \cap W_{\mu}}$. The preceding considerations show that the diagram

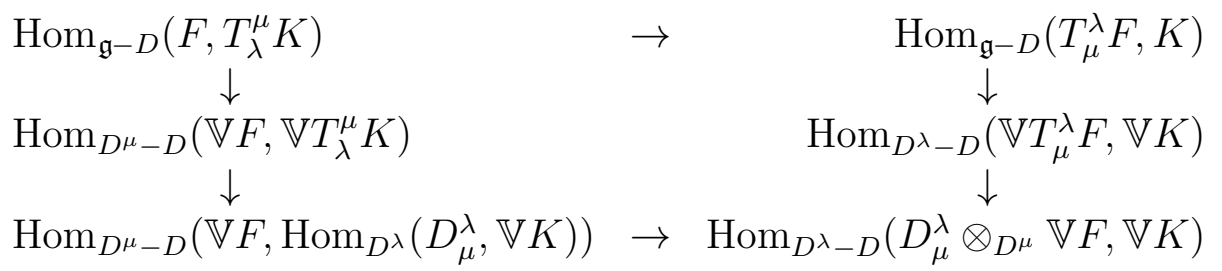

commutes, if we define both lower verticals by the isomorphisms just introduced and the horizonals by the adjunctions. In this diagram all morphisms with the possible exception of both upper verticals are obviously isomorphisms. Thus if the right upper vertical is an isomorphism, then the left 
upper vertical as well. If in other words our claim holds for $K$, then it also holds for $T_{\lambda}^{\mu} K$. Thus it suffices to check it for $K$ a deformed simple Verma. Working down through a Verma flag, we may even assume $F$ to be a direct sum of copies of this simple Verma. In this case the first claim is obvious. The second claim is shown in the same way.

\section{Geometrical arguments}

6.1. Let gMod- $A$ denote the category of graded right modules over a graded ring $A$. Let $\operatorname{Der}_{G}(X)$ resp. $\operatorname{Der}_{G}^{+}(X)$ denote the equivariant resp. bounded below equivariant derived category corresponding to a complex algebraic variety $X$ with the action of a complex algebraic group $G$ and let $\operatorname{Der}_{G}(\mathcal{F}, \mathcal{G})$ denote the morphisms in these categories, as explained in [BL94]. Here and in what follows we will always take cohomology with complex coefficients.

6.2. Let $X$ be a complex algebraic variety with the action of an algebraic group $B$. Let $X=\coprod_{a \in A} X_{a}$ be a stratification into irreducible locally closed smooth $B$-stable subvarieties such that the closure of each stratum is a union of strata. Let $|a|$ denote the dimension of $X_{a}$ and $\mathcal{C}_{a}=\underline{X_{a}}[|a|]$ the "constant perverse sheaf" in $\operatorname{Der}_{B}\left(X_{a}\right)$. Let further $j_{a}: X_{a} \hookrightarrow X$ denote the inclusion. Let now $\mathcal{F}, \mathcal{G} \in \operatorname{Der}_{B}(X)$ be given with the property, that for all $a \in A$ we have finite direct sum decompositions

$$
\begin{aligned}
j_{a}^{*} \mathcal{F} & \cong \bigoplus_{\nu} f_{a}^{\nu} \mathcal{C}_{a}[\nu] \\
j_{a}^{!} \mathcal{G} & \cong \bigoplus_{\nu} g_{a}^{\nu} \mathcal{C}_{a}[\nu]
\end{aligned}
$$

in $\operatorname{Der}_{B}\left(X_{a}\right)$ for suitable $f_{a}^{\nu}, g_{a}^{\nu} \in \mathbb{N}$. If under these assumptions we also have $f_{a}^{\nu}=0=g_{a}^{\nu}$ for $\nu+|a|$ odd and $H_{B}^{\nu}\left(X_{a}\right)=0$ for $\nu$ odd, then taking the equivariant hypercohomology $\mathbb{H}_{B}$ induces for all $*$ an injection

$$
\operatorname{Der}_{B}(\mathcal{F}, \mathcal{G}[*]) \hookrightarrow \operatorname{Hom}\left(\mathbb{H}_{B} \mathcal{F}, \mathbb{H}_{B} \mathcal{G}\right)
$$

and the dimensions of the homogeneous components on the left are given by the formula

$$
\operatorname{dim} \operatorname{Der}_{B}(\mathcal{F}, \mathcal{G}[n])=\sum_{\nu-\mu+k=n, a \in A} f_{a}^{\nu} g_{a}^{\mu} \operatorname{dim} H_{B}^{k}\left(X_{a}\right)
$$

The proof is completely analogous to the proof of Proposition 3 on page 404 of [Soe01] and we shall not repeat it here.

6.3. Let $G \supset P=P_{\iota} \supset B \supset T$ be a semisimple complex algebraic group, a parabolic, a Borel and a maximal torus. Let $W_{\iota} \subset W$ be the Weyl groups of 
$P \subset G$ and $L \supset T$ the Levi of $P$ above $T$. We let $B \times P$ act on $G$ by the rule $(b, p) g=b g p^{-1}$. From [BL94 we deduce that the equivariant cohomology $H_{B \times P}^{*}(G)$ becomes under restriction a quotient of $H_{B \times P}^{*}(\mathrm{pt})=H_{T \times L}^{*}(\mathrm{pt})=$ $R \otimes_{\mathbb{C}} R^{\iota}$ for $R=\mathbb{C}[\operatorname{Lie} T]$ the ring of regular functions on Lie $T$, graded by the condition that linear forms should be homogeneous of degree two, and $R^{\iota}$ the invariants of $W_{\iota}$ in $R$. Again using [BL94] we get in this way a canonical isomorphism

$$
c: R \otimes_{R^{W}} R^{\iota} \stackrel{\sim}{\rightarrow} H_{B \times P}^{*}(G)
$$

Thus the equivariant hypercohomology

$$
\mathbb{H}_{B \times P}^{*}: \operatorname{Der}_{B \times P}^{+}(G) \rightarrow \text { gMod- } H_{B \times P}^{*}(\mathrm{pt})
$$

defines under our identification of the equivariant cohomology ring and the identification $\operatorname{Der}_{B \times P}^{+}(G) \cong \operatorname{Der}_{B}^{+}(G / P)$ a functor to $\mathbb{Z}$-graded $R$ - $R^{\iota}$-bimodules

$$
\mathbb{H}_{B}=\mathbb{H}_{B}^{*}: \operatorname{Der}_{B}^{+}(G / P) \rightarrow R \text {-gMod- } R^{\iota}
$$

Now we consider in $\operatorname{Der}_{B}^{+}(G / P)$ for $x \in W / W_{\iota}$ the intersection cohomology complex $\mathcal{I C}_{x}$ of the closure of $B x P / P$. Let $\mathcal{C}_{y}$ be the constant perverse sheaf on $B y B / P$, which is concentrated in degree $-l(y)$ as a complex of ordinary sheaves, and let $j_{y}: B y B / P \hookrightarrow G / P$ denote the embedding.

Theorem 6.4. The functor $\mathbb{H}_{B}$ is fully faithful on morphisms $\mathcal{I C}_{x} \rightarrow j_{y *} \mathcal{C}_{y}[n]$ and $j_{x !} \mathcal{C}_{y} \rightarrow \mathcal{I C}_{y}[n]$ and $\mathcal{I C}_{x} \rightarrow \mathcal{I C}_{y}[n]$ in $\operatorname{Der}_{B}^{+}(G / P)$.

Proof. In Gin91 the corresponding statement is proven for nonequivariant cohomology and the case $\mathcal{I C} \rightarrow \mathcal{I C}$, but in a more general setup. In [Soe01, Proposition 2, page 402 this is proven for homomorphisms $\mathcal{I C}_{x} \rightarrow \mathcal{I C}_{y}[n]$ and $P=B$. I will now explain in which sense the proof given there up to some rather minor modifications also proves this more general case. First we restrict to the case $P=B$. By Lemma 6 on page 405 of Soe01 in connection with 6.1 the functor of the lemma is faithful and the dimensions of the Hom spaces in question are known. By [Soe07] however we also know the dimensions of the Hom spaces in the image and thus we may finish the argument with a comparision of dimensions. More precisely we get with 6.1 the formula

$$
\operatorname{dim}_{\mathbb{C}} \operatorname{Der}_{B}\left(\mathcal{I} \mathcal{C}_{x}, j_{y *} \mathcal{C}_{y}[n]\right)=\sum_{k+i=n} n_{y, x}^{i} \operatorname{dim}_{\mathbb{C}} H_{B}^{k}(B y B / B)
$$

Here the $n_{y, x}^{i}$ are the coefficients of the Kazhdan-Lusztig polynomials and we have more precisely

$$
\sum_{y, i} n_{y, x}^{i} q^{-i / 2} \tilde{T}_{y}=C_{x}^{\prime}
$$


in Lusztig's notation alias $\sum_{y, i} n_{y, x}^{i} v^{i} H_{y}=\underline{H}_{x}$ in the notations of [Soe97b]. On the other hand in [Soe01], Lemma 5, page 402 it is shown for $P=B$, that the $\mathbb{H}_{B} \mathcal{I} \mathcal{C}_{x}$ are just the special bimodules

$$
\mathbb{H}_{B} \mathcal{I} \mathcal{C}_{x} \cong B_{x}
$$

which I consider in [Soe92] and [Soe07]. Now we recall the graded bimodule $R_{y}$ from [Soe07], which is free of rank one from the left and the right with the same generator $1_{y}$ in degree zero and the property $r 1_{y}=1_{y} r^{y}$ for $r^{y}=y^{-1}(r)$, and we recall its shifted versions $\Delta_{y}=R_{y}[-l(y)]$ and $\nabla_{y}=R_{y}[l(y)]$. One shows easily $\mathbb{H}_{B} j_{y *} \mathcal{C}_{y} \cong \nabla_{y}$ and $\mathbb{H}_{B} j_{y !} \mathcal{C}_{y} \cong \Delta_{y}$. Thus we need to establish the equality of dimenisons

$$
\operatorname{dim}_{\mathbb{C}} \operatorname{Der}_{B}\left(\mathcal{I C}_{x}, j_{y *} \mathcal{C}_{y}[n]\right)=\operatorname{dim}_{\mathbb{C}} \operatorname{gMod}_{R-R}\left(B_{x}, \nabla_{y}[n]\right) .
$$

But by [Soe07], Theorem 5.15 the space $\operatorname{Mod}_{R-R}\left(B_{x}, \nabla_{y}\right)$ is graded free as a right $R$-module, and if we let $h_{y, x}^{i}$ be the number of generators needed in degree $i$, then Theorem 5.3 of [Soe07] gives in the Hecke algebra

$$
C_{x}^{\prime}=\sum_{y \in W} h_{y, x}^{i} q^{-1 / 2} \tilde{T}_{y}=\sum_{y \in W} h_{y, x}^{i} v^{i} H_{y}
$$

in the notations of Lusztig resp. of [Soe97b]. In other words we get $h_{y, x}^{i}=n_{y, x}^{i}$, and since $H_{B}^{*}(B y B / B) \cong R$ we deduce the claimed equality of dimensions in every degree. The second case follows dually and thus in case $P=B$ we have completely established the Lemma. In general full faithfulness of our functor is deduced in the same way, but for the equality of dimensions we need to work a little more. Here we only treat the cases $\mathcal{I C} \rightarrow \mathcal{I C}$ and $\mathcal{I C} \rightarrow \mathcal{C}$, the remaining case is dual. Let $\pi: G / B \rightarrow G / P$ denote the projection, so that we get $\mathbb{H}_{B} \pi_{*} \mathcal{G} \cong \operatorname{res}_{R-R}^{R-R^{\iota}} \mathbb{H}_{B} \mathcal{G}$ and $\mathcal{H}_{B} \pi^{*} \mathcal{F} \cong \mathbb{H}_{B} \mathcal{F} \otimes_{R^{\iota}} R$. This leads to a commutative diagramm

$$
\begin{array}{ccc}
\operatorname{Der}_{B}\left(\mathcal{F}, \pi_{*} \mathcal{G}[n]\right) & \stackrel{\sim}{\longrightarrow} & \operatorname{Der}_{B}\left(\pi^{*} \mathcal{F}, \mathcal{G}[n]\right) \\
\downarrow & \downarrow & \downarrow \\
\operatorname{gMod}_{R-R^{\iota}}\left(\mathbb{H}_{B} \mathcal{F}, \mathbb{H}_{B} \pi_{*} \mathcal{G}[n]\right) & & \operatorname{gMod}_{R-R}\left(\mathbb{H}_{B} \pi^{*} \mathcal{F}, \mathbb{H}_{B} \mathcal{G}[n]\right) \\
\| & & \| \\
\operatorname{gMod}_{R-R^{\iota}}\left(\mathbb{H}_{B} \mathcal{F}, \operatorname{res}_{R-R}^{R-R^{\iota}} \mathbb{H}_{B} \mathcal{G}[n]\right) & \stackrel{\sim}{\longrightarrow} \operatorname{gMod}_{R-R}\left(\mathbb{H}_{B} \mathcal{F} \otimes_{R^{\iota}} R, \mathbb{H}_{B} \mathcal{G}[n]\right)
\end{array}
$$

and with the right upper vertical the left upper vertical must be an isomorphism, too. Thus the cases $\mathcal{I C} \rightarrow \mathcal{I C}$ and $\mathcal{I C} \rightarrow \mathcal{C}$ follow for general $P$ from the case $P=B$. 


\section{$7 \quad$ Singular bimodules}

7.1. Let $\mathcal{W}$ be a finite group of automorphisms of a finite dimensional affine space $E$ over $\mathbb{Q}$, which is generated by reflections, and let $\mathcal{S} \subset \mathcal{W}$ be a choice of simple reflections. Let $R$ denote the regular functions on the space of translations, graded by the rule, that linear functions are homogeneous of degree two. Then by [Soe07] there exist well-defined up to isomorphism $\mathbb{Z}$-graded $R$-bimodules $B_{x}=B_{x}(\mathcal{W})=B_{x}(\mathcal{W}, \mathcal{S}, E) \in R$-gMod- $R$ such that we have

1. The $B_{x}$ are indecomposable.

2. For $e$ the neutral element we have $B_{e}=R$.

3. If $s \in \mathcal{S}$ is a simple reflection with $x s>x$, then there is a decomposition

$$
B_{x} \otimes_{R^{s}} R[1] \cong B_{x s} \oplus \bigoplus_{l(y) \leq l(x)} m(y) B_{y}
$$

for suitable multiplicities $m(y) \in \mathbb{N}$.

Following [Soe07] the rings of endomorphisms of degree zero of these bimodules consist just of scalars, in particular our bimodules stay indecomposable when we extend scalars. Now let $\mathcal{S}_{\iota} \subset \mathcal{S}$ be a subset of the set of simple reflections, $\mathcal{W}_{\iota}=\left\langle\mathcal{S}_{\iota}\right\rangle \subset \mathcal{W}$ the subgroup generated by it, $w_{\iota} \in \mathcal{W}_{\iota}$ its longest element and $R^{\iota}$ the subring of $\mathcal{W}_{\iota}$-invariants. Then under the same assumptions we claim:

Lemma 7.2. For every coset $\bar{x} \in \mathcal{W} / \mathcal{W}_{\iota}$ there exists one and only one $\mathbb{Z}$ graded $R$ - $R^{\iota}$-bimodule $B_{\bar{x}}^{\iota}=B_{\bar{x}}^{\iota}\left(\mathcal{W}, \mathcal{W}_{\iota}\right) \in R$-gMod- $R^{\iota}$ with the property that for $x \in \mathcal{W}$ the longest representative of the coset $\bar{x}$ we have

$$
\operatorname{res}_{R-R}^{R-R^{\iota}} B_{x} \cong \bigoplus_{z \in \mathcal{W}_{\iota}} B_{\bar{x}}^{\iota}\left[l\left(w_{\iota}\right)-2 l(z)\right]
$$

Proof. Without restriction of generality we may assume that $\mathcal{W}$ admits only one fixed point. Since by assumption it is a rational and thus crystallographic reflection group, we then find $G \supset B \supset T$ a complex semisimple algebraic group $G$ with Borel $B$ and maximal torus $T$ and Coxeter system $(\mathcal{W}, \mathcal{S})$. If we identify in a $\mathcal{W}$-equivariant way $H_{T}^{2}(\mathrm{pt} ; \mathbb{Q})$ and the homogeneous component $R^{2}$ of $R$, then as we discussed already in the proof of 6.4 there exists an isomorphism of $\mathbb{Z}$-graded $R$-bimodules

$$
B_{x} \cong \mathbb{H}_{B} \mathcal{I C}(\overline{B x B / B})
$$


If $P=P_{\iota}$ is a parabolic with $G \supset P \supset B$, then the decomposition theorem of [BL94] applied to the projection $p: G / B \rightarrow G / P$, shows for $x$ maximal in its $\mathcal{W}_{\iota}$-coset the existence of a decomposition

$$
p_{*} \mathcal{I C}(\overline{B x B / B}) \cong \bigoplus_{z \in \mathcal{W}_{\iota}} \mathcal{I C}(\overline{B x P / P})\left[l\left(w_{\iota}\right)-2 l(z)\right]
$$

in $\operatorname{Der}_{B}^{+}(G / P)$. On the other hand we have $\mathbb{H}_{B} \circ p_{*}=\operatorname{res}_{R-R}^{R-R^{\imath}} \circ \mathbb{H}_{B}$ and the $\mathbb{H}_{B} \mathcal{I C}(\overline{B x P / P})$ are indecomposable as graded $R$ - $R^{\iota}$-bimodules, since by 6.4 the scalars are their only endomorphisms of degree $\leq 0$.

\section{The bimodules for tilting modules}

8.1. Given $y \in W$ let $\hat{S}_{y}$ denote the bimodule, which from the left is free over $\hat{S}$ of rank one with basis say $1_{y}$, but from the right has the action $r 1_{y}=1_{y} r^{y}$ of $\hat{S}$. Given a bimodule $B$ for two commutative rings let $\tilde{B}$ denote the bimodule which we get by interchanging the right and the left action.

Theorem 8.2. Let $\lambda \in \mathfrak{h}_{\text {dom }}^{*}$ and let $W_{\lambda} \subset W_{\bar{\lambda}} \subset W$ be as in 5.6. Given $\bar{x} \in W_{\bar{\lambda}} / W_{\lambda}$ we have for the deformation of the indecomposable tilting module with highest weight $w_{\bar{\lambda}} \bar{x} \cdot \lambda$ the formula

$$
\mathbb{V} K_{\hat{S}}\left(w_{\bar{\lambda}} \bar{x} \cdot \lambda\right) \cong \widetilde{B}_{\bar{x}}^{\lambda} \otimes_{S} \hat{S}_{w_{\bar{\lambda}}}
$$

Remark 8.3. Our bimodules $B_{\bar{x}}^{\iota}$ are graded free of finite rank over $R$, thus $\hat{R} \otimes_{R} B_{\bar{x}}^{\iota}$ is just the completion along the grading of our original bimodule $B_{\bar{x}}^{\iota}$ and in particular this completion admits a right action of $\hat{R}^{\iota}$.

Proof. As is well-known and explained in Remark 7.2.2 of [Soe97b], an indecomposable tilting module stays indecomposable upon translation out of the walls. More precisely for $\lambda, \mu \in \mathfrak{h}_{\text {dom }}^{*}$ with $\lambda+X=\mu+X$ and $W_{\mu}=1$ and $x \in W_{\bar{\lambda}}$ maximal in its coset $x W_{\lambda}$ we have

$$
T_{\lambda}^{\mu} K\left(w_{\bar{\lambda}} x \cdot \lambda\right)=K\left(w_{\bar{\mu}} x \cdot \mu\right)
$$

Since $T_{\mu}^{\lambda} T_{\lambda}^{\mu}$ is a sum of $\left|W_{\lambda}\right|$ copies of the identity functor by [BG80, we may for the proof restrict to the case $\lambda$ regular. If $x=s t \ldots r$ is a reduced decomposition by simple reflections of $W_{\bar{\lambda}}$, we may characterize $K_{\hat{S}}\left(w_{\bar{\lambda}} x \cdot \lambda\right)$ inductively as the indecomposable summand of

$$
\vartheta_{r} \ldots \vartheta_{t} \vartheta_{s} \Delta_{\hat{S}}\left(w_{\bar{\lambda}} \cdot \lambda\right)
$$


not isomorphic to any $K_{\hat{S}}\left(w_{\bar{\lambda}} y \cdot \lambda\right)$ for $y<x$. Applying $\mathbb{V}$ we get from this the indecomposable summand of

$$
\hat{S} \otimes_{\hat{S}^{r}} \hat{S} \ldots \otimes_{\hat{S}^{t}} \hat{S} \otimes_{\hat{S}^{s}} \hat{S}_{w_{\bar{\lambda}}}
$$

which didn't appear already before. But by definition of our special bimodules this is precisely $\widetilde{B}_{x} \otimes_{S} \hat{S}_{w_{\bar{\lambda}}}$.

\section{Restricting the group action}

9.1. For $G$ a complex connected algebraic group we let $A_{G}=H^{*}(B G)$ be the cohomology ring of its classifying space. If $X$ is a complex algebraic $G$-variety and $\mathcal{F}, \mathcal{G} \in \operatorname{Der}_{G}^{+}(X)$ are objects of the equivariant derived category, we may form the graded $A_{G}$-module

$$
\operatorname{Der}_{G}(\mathcal{F}, \mathcal{G}[*])=\bigoplus_{n} \operatorname{Der}_{G}(\mathcal{F}, \mathcal{G}[n])
$$

Proposition 9.2. Let $G \supset H$ be a connected complex algebraic group and a connected closed subgroup. Let $X$ be an algebraic $G$-variety and let $\mathcal{F}, \mathcal{G} \in$ $\operatorname{Der}_{G}(X)$ be constructible complexes. If $\operatorname{Der}_{G}(\mathcal{F}, \mathcal{G}[*])$ is graded free over $A_{G}$, then the obvious map induces a bijection

$$
A_{H} \otimes_{A_{G}} \operatorname{Der}_{G}(\mathcal{F}, \mathcal{G}[*]) \stackrel{\sim}{\rightarrow} \operatorname{Der}_{H}(\mathcal{F}, \mathcal{G}[*])
$$

Proof. We consider the constant map $k: X \rightarrow$ pt and the fully faithful functor $\gamma_{G}: \operatorname{Der}_{G}^{\mathrm{c}}(\mathrm{pt}) \rightarrow A_{G}$-dgDer from [BL94], 12.4.6 and recall

$$
\operatorname{Der}_{G}(\mathcal{F}, \mathcal{G}[*])=H^{*} \gamma_{G} k_{*} \operatorname{Hom}(\mathcal{F}, \mathcal{G})
$$

where we form $\operatorname{Hom}(\mathcal{F}, \mathcal{G})$ in $\operatorname{Der}_{G}^{+}(X)$ and $k_{*}$ means the direct image landing in $\operatorname{Der}_{G}^{+}(\mathrm{pt})$. If this now is a free $A_{G}$-module, then $\gamma_{G} k_{*} \operatorname{Hom}(\mathcal{F}, \mathcal{G})$ is already quasiisomorphic to its cohomology and this cohomology is homotopy projective in $A_{G}$-dgMod. With the derived functor $A_{H} \otimes_{A_{G}}^{L}: A_{G}$-dgDer $\rightarrow$ $A_{H}$-dgDer we have by [BL94], 12.7.1 furthermore canonically

$$
\left(A_{H} \otimes_{A_{G}}^{L}\right) \circ \gamma_{G}=\gamma_{H} \circ \operatorname{res}_{G}^{H}
$$

and for homotopy projective objects $M \in A_{G}$-dgMod we have in addition

$$
A_{H} \otimes_{A_{G}}^{L} M=A_{H} \otimes_{A_{G}} M
$$

Since $k_{*}$ and Hom commute with the restriction of the group action, this shows the Proposition. 


\section{Proof of the main theorem 4.4}

10.1. Given a ring $R$, a ring homomorphism $R \rightarrow \mathbb{C}[[v]]$, three $R$-modules $H, H^{\prime}, H^{\prime \prime}$ and an $R$-bilinear map

$$
\varphi: H \times H^{\prime} \rightarrow H^{\prime \prime}
$$

we may introduce on $\mathbb{C}[[v]] \otimes_{R} H$ a filtration by the rule

$$
F^{i}\left(\mathbb{C}[[v]] \otimes_{R} H\right)=\left\{\begin{array}{l|l}
h & \begin{array}{l}
\varphi\left(h, h^{\prime}\right) \in v^{i} \mathbb{C}[[v]] \otimes_{R} H^{\prime \prime} \\
\text { for all } h^{\prime} \in \mathbb{C}[[v]] \otimes_{R} H^{\prime}
\end{array}
\end{array}\right\}
$$

and get also an induced filtration on $\mathbb{C} \otimes_{R} H$, whose subquotients we denote $\bar{F}^{i}\left(\mathbb{C} \otimes_{R} H\right)$.

10.2. If for example $\hat{S}$ is the completion of $S=\mathcal{O}\left(\mathfrak{h}^{*}\right)$ along the natural grading and $\hat{S} \rightarrow \mathbb{C}[[v]]$ is the restriction to the line $\mathbb{C} \rho$ as in 4.2 , then the pairing given by composition

$$
\begin{aligned}
\operatorname{Hom}_{\mathfrak{g}-\hat{S}}\left(\Delta_{\hat{S}}(\lambda), K_{\hat{S}}(\mu)\right) & \times \operatorname{Hom}_{\mathfrak{g}-\hat{S}}\left(K_{\hat{S}}(\mu), \nabla_{\hat{S}}(\lambda)\right) \\
& \rightarrow \operatorname{Hom}_{\mathfrak{g}-\hat{S}}\left(\Delta_{\hat{S}}(\lambda), \nabla_{\hat{S}}(\lambda)\right)
\end{aligned}
$$

leads to the Andersen filtration 4.2 on the spaces $\operatorname{Hom}_{\mathfrak{g}}(\Delta(\lambda), K(\mu))$, which by 2.13 may be identified with $\operatorname{Hom}_{\mathfrak{g}-\hat{S}}\left(\Delta_{\hat{S}}(\lambda), K_{\hat{S}}(\mu)\right) \otimes_{\hat{S}} \mathbb{C}$. Certainly there also exists $p \in \hat{S}$ such that $\mathbb{V}$ induces an isomorphism

$$
\operatorname{Hom}_{\mathfrak{g}-\hat{S}}\left(\Delta_{\hat{S}}(\lambda), \nabla_{\hat{S}}(\lambda)\right) \stackrel{\sim}{\rightarrow} p \operatorname{Hom}_{\hat{S}}\left(\mathbb{V} \Delta_{\hat{S}}(\lambda), \mathbb{V} \nabla_{\hat{S}}(\lambda)\right)
$$

and with this $p$ we may reformulate our pairing as in the pairing given by the composition

$$
\begin{array}{r}
\operatorname{Hom}_{\hat{S}^{\lambda}-\hat{S}}\left(\mathbb{V} \Delta_{\hat{S}}(\lambda), \mathbb{V} K_{\hat{S}}(\mu)\right) \times \operatorname{Hom}_{\hat{S}^{\lambda}-\hat{S}}\left(\mathbb{V} K_{\hat{S}}(\mu), \mathbb{V} \nabla_{\hat{S}}(\lambda)\right) \\
\rightarrow p \operatorname{Hom}_{\hat{S}^{\lambda}-\hat{S}}\left(\mathbb{V} \Delta_{\hat{S}}(\lambda), \mathbb{V} \nabla_{\hat{S}}(\lambda)\right)
\end{array}
$$

Here a possible $p$ may be determined by the condition that our pairing in case $\lambda=\mu$ must lead to a surjection. Now we change parameters, choose $\lambda \in \mathfrak{h}_{\text {dom }}^{*}$ and put $\lambda_{\bar{x}}=w_{\bar{\lambda}} \bar{x} \cdot \lambda$ for $\bar{x} \in W_{\bar{\lambda}} / W_{\lambda}$. To simplify we further introduce a variant $\widetilde{\mathbb{V}}$ of $\mathbb{V}$ by putting

$$
\widetilde{\mathbb{V}} M=\hat{S}_{w_{\bar{\lambda}}} \otimes_{\hat{S}} \widetilde{\mathbb{V} M}
$$

such that 8.2 becomes $\widetilde{\mathbb{V}} K_{\hat{S}}\left(\lambda_{\bar{x}}\right) \cong \hat{B}_{\bar{x}}^{\lambda}$, the hat meaning completion along the grading. With less effort one may also check

$$
\widetilde{\mathbb{V}} \Delta_{\hat{S}}\left(\lambda_{\bar{y}}\right) \cong \widetilde{\mathbb{V}} \nabla_{\hat{S}}\left(\lambda_{\bar{y}}\right) \cong \hat{S}_{\bar{y}}^{\lambda}
$$


in $\hat{S}$-mod- $\hat{S}^{\lambda}$, where again we mean the bimodule which is $\hat{S}$ from the left but has the $\bar{y}$-twisted action $r 1_{y}=1_{y} r^{y}$ of $\hat{S}^{\lambda}$ from the right. If we replace $\mathbb{V}$

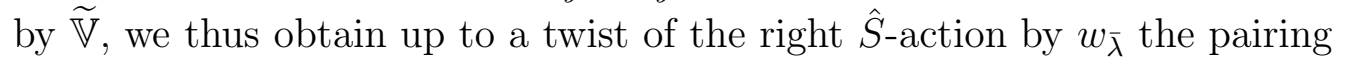

$$
\begin{array}{r}
\operatorname{Hom}_{\hat{S}-\hat{S}^{\lambda}}\left(\hat{S}_{\bar{y}}^{\lambda}, \hat{B}_{\bar{x}}^{\lambda}\right) \times \operatorname{Hom}_{\hat{S}-\hat{S}^{\lambda}}\left(\hat{B}_{\bar{x}}^{\lambda}, \hat{S}_{\bar{y}}^{\lambda}\right) \\
\rightarrow p_{1} \operatorname{Hom}_{\hat{S}-\hat{S}^{\lambda}}\left(\hat{S}_{\bar{y}}^{\lambda}, \hat{S}_{\bar{y}}^{\lambda}\right)
\end{array}
$$

of $\hat{S}$-modules and our filtration corresponds to the filtration we get here when we change $\hat{S} \rightarrow \mathbb{C}[[v]]$ by twisting it with $w_{\bar{\lambda}}$. Here $p_{1}$ denotes the image of $p$ under $w_{\bar{\lambda}}$. Since the choice of $p_{1}$ is only sensible up to units of $\hat{S}$, we may choose $p_{1}$ already before completion and the corresponding pairing "before completion"

$$
\begin{array}{r}
\operatorname{Hom}_{S-S^{\lambda}}\left(S_{\bar{y}}^{\lambda}, B_{\bar{x}}^{\lambda}\right) \times \operatorname{Hom}_{S-S^{\lambda}}\left(B_{\bar{x}}^{\lambda}, S_{\bar{y}}^{\lambda}\right) \\
\rightarrow p_{1} \operatorname{Hom}_{S-S^{\lambda}}\left(S_{\bar{y}}^{\lambda}, S_{\bar{y}}^{\lambda}\right)
\end{array}
$$

leads to the same filtered $\mathbb{C}$-space in the end. This pairing we now interpret geometrically.

10.3. If $X \subset \mathfrak{h}^{*}$ denotes the lattice of integral weights, we find a pair $G^{\vee} \supset T^{\vee}$ consisting of a reductive connected complex algebraic group with a maximal torus such that $X=X\left(T^{\vee}\right)$ is its group of one-parameter subgroups and that for the Weyl group we have $W\left(G^{\vee}, T^{\vee}\right)=W_{\bar{\lambda}}$. In $G^{\vee}$ we then choose a Borel $B^{\vee}$ for $\mathcal{S} \cap W_{\bar{\lambda}}$ and a parabolic $P^{\vee} \supset B^{\vee}$ for $W_{\lambda}$. If now

$$
\mathcal{I C}_{\bar{x}}=\mathcal{I C}\left(\overline{B^{\vee} \bar{x} P^{\vee} / P^{\vee}}\right)
$$

denotes the intersection homology complex of the corresponding Schubert variety and $\mathcal{C}_{\bar{y}}$ the constant perverse sheaf on $B^{\vee} \bar{y} P^{\vee} / P^{\vee}$, we have $B_{\bar{x}}^{\lambda} \cong$ $\mathbb{H}_{B^{\vee}} \mathcal{I C}_{\bar{x}}$ and our pairing "before completion" from the end of the previous remark can be interpreted with the help of 6.4 as the pairing given by composition

$$
\begin{array}{r}
\operatorname{Der}_{B^{\vee}}\left(j_{\bar{y} !} \mathcal{C}_{\bar{y}}, \mathcal{I C}_{\bar{x}}[*]\right) \times \operatorname{Der}_{B^{\vee}}\left(\mathcal{I C}_{\bar{x}}, j_{\bar{y} *} \mathcal{C}_{\bar{y}}[*]\right) \\
\rightarrow \operatorname{Der}_{B^{\vee}}\left(j_{\bar{y} !} \mathcal{C}_{\bar{y}}, j_{\bar{y}} \mathcal{C}_{\bar{y}}[*]\right)
\end{array}
$$

Here we do not need a $p$-factor on the right hand side, since restriction to the big cell shows that for $\bar{x}=\bar{y}$ our pairing gives a surjection. The question is thus, which filtered vector space this pairing of $A_{B^{\vee} \text {-modules leads to under }}$ the homomorphism $A_{B^{\vee}} \rightarrow \mathbb{C}[v]$ coming from the embedding $\mathbb{C}^{\times} \hookrightarrow T^{\vee}$ with parameter $w_{\bar{\lambda}} \rho$. But by 9.2 this specialization leads us to the composition pairing

$$
\begin{array}{r}
\operatorname{Der}_{\mathbb{C}^{\times}}\left(j_{\bar{y} !} \mathcal{C}_{\bar{y}}, \mathcal{I C}_{\bar{x}}[*]\right) \times \operatorname{Der}_{\mathbb{C}^{\times}}\left(\mathcal{I C}_{\bar{x}}, j_{\bar{y} *} \mathcal{C}_{\bar{y}}[*]\right) \\
\rightarrow \operatorname{Der}_{\mathbb{C}^{\times}}\left(j_{\bar{y} !} \mathcal{C}_{\bar{y}}, j_{\bar{y}} \mathcal{C}_{\bar{y}}[*]\right)
\end{array}
$$


Let now $\bar{y}$ denote the point $\bar{y} P^{\vee}$ of $G^{\vee} / P^{\vee}$. For a suitable product $U$ of root subgroups of $G^{\vee}$ the multiplication $u \mapsto u \bar{y}$ defines an embedding $U \hookrightarrow$ $G^{\vee} / P^{\vee}$, whose image is a cell transversal to $B^{\vee} \bar{y} P^{\vee} / P^{\vee}$ and is contracted by $\mathbb{C}^{\times}$to $\bar{y}$. If we put $Z=U \bar{y} \cap \overline{B^{\vee} \bar{x} P^{\vee} / P^{\vee}}$, then $Z$ is contracted by $\mathbb{C}^{\times}$ to $\bar{y}$, and if $a: Z \hookrightarrow G^{\vee} / P^{\vee}$ denotes the embedding, the restriction to $Z$ will not change our pairing. If we now put $d=\operatorname{dim} B^{\vee} \bar{y} P^{\vee} / P^{\vee}$ and let $i: \mathrm{pt} \hookrightarrow Z$ be the embedding of $\bar{y}$ and pt the constant sheaf on a point, we get $a^{*} j_{\bar{y} !} \mathcal{C}_{\bar{y}} \cong i_{*} \underline{\operatorname{pt}}[d] \cong a^{*} j_{\bar{y}_{*}} \mathcal{C}_{\bar{y}}$ and $a^{*} \mathcal{I} \mathcal{C}_{\bar{x}} \cong \mathcal{I C}[d]$ will be the shifted intersection cohomology complex $\mathcal{I C}=\mathcal{I C}(Z)$ of $Z$ and our pairing gets transformed to the composition pairing

$$
\begin{array}{r}
\operatorname{Der}_{\mathbb{C}^{\times}}\left(i_{*} \underline{\mathrm{pt}}, \mathcal{I C}[*]\right) \times \operatorname{Der}_{\mathbb{C}^{\times}}\left(\mathcal{I C}, i_{*} \underline{\mathrm{pt}}[*]\right) \\
\rightarrow \operatorname{Der}_{\mathbb{C}^{\times}}\left(i_{*} \underline{\mathrm{pt}}, i_{*} \underline{\mathrm{pt}}[*]\right)
\end{array}
$$

Now we may identify the first of these paired modules with $H_{\mathbb{C}^{\times}}\left(i^{\prime} \mathcal{I C}\right)$ and the second with the dual of $H_{\mathbb{C}^{\times}}\left(i^{*} \mathcal{I C}\right)$ and thus our pairing leads to the same filtered space as the embedding of free $\mathbb{C}[v]$-modules $H_{\mathbb{C}^{\times}}\left(i^{!} \mathcal{I C}\right) \hookrightarrow$ $H_{\mathbb{C}^{\times}}\left(i^{*} \mathcal{I C}\right)$. But in this situation the "fundamental example" of section 14 of BL94 just means that the cokernel of this embedding may be identified with the intersection cohomology of the projective variety $\bar{Z}=(Z \backslash\{\bar{y}\}) / \mathbb{C}^{\times}$ shifted by one, with $I C(\bar{Z})[1]$ viewed as a $\mathbb{C}[v]$-module in such a way, that $v$ acts as Lefschetz operator, thus leading to a short exact sequence of $\mathbb{Z}$-graded $\mathbb{C}[v]$-modules

$$
H_{\mathbb{C}^{\times}}\left(i^{!} \mathcal{I C}\right) \hookrightarrow H_{\mathbb{C}^{\times}}\left(i^{*} \mathcal{I C}\right) \rightarrow I C(\bar{Z})[1]
$$

More precisely, this goes as follows: One starts with the decomposition of $Z$ into the center of the contraction pt and its open complement $Z_{0}$ and denotes the inclusions by $i$ and $j$ and writes the Gysin triangle in the equivariant derived category pushed down by a map $p$ to a point

$$
p_{*} i_{!} i \mathfrak{I} \mathcal{C} \rightarrow p_{*} \mathcal{I C} \rightarrow p_{*} j_{*} j^{*} \mathcal{I C} \stackrel{[1]}{\rightarrow}
$$

This triangle in $\operatorname{Der}_{\mathbb{C} \times}(\mathrm{pt})$ can by Theorem 14.2 in loc.cit. be identified with a triangle in the derived category of dg-modules $\mathbb{C}[v]$-dgDer over $\mathbb{C}[v]=$ $H_{\mathbb{C}^{\times}}^{*}(\mathrm{pt})$ written

$$
\left(\tau_{\geq 0} M\right)[-1] \rightarrow \tau_{<0} M \rightarrow M \stackrel{[1]}{\rightarrow}
$$

in the notation of loc.cit. However at the end of the proof of this Theorem following loc.cit. Lemma 14.15 this is further rewritten as a short exact sequence of graded $\mathbb{C}[v]$-modules and $M$ is identified with $I C(\bar{Z})[1]$ by a remark preceding loc.cit. 14.5 labeled 13.4, since in our case $G$ is trivial, whereas $\left(\tau_{\geq 0} M\right)[-1]$ is identified with the costalk by loc.cit. Theorem 14.2(i) 
and this costalk with its cohomology by loc.cit. 14.3(i). The middle part of our Gysin sequence finally may also be interpreted as the stalk at the center of the contraction, $p_{*} \mathcal{I C} \cong i^{*} \mathcal{I C}$, as explained in [Spr84], section 3, and again this stalk when written as a dg-module can be identified with its cohomology by BL94 14.3(i'). In this way we see that BL94] indeed leads to the short exact sequence of $\mathbb{C}[v]$-modules I claimed. The hard Lefschetz from [BBD82] 6.2.10 now tells us, that as a graded module over $\mathbb{C}[v]$ our intersection cohomology $I C(\bar{Z})$ is a direct sum of truncated polynomial rings graded in such a way they are selfdual, in formulas a direct sum of graded modules of the form $\left(\mathbb{C}[v] /\left(v^{i+1}\right)\right)[i]$. Furthermore we know from the description of our sequence by truncation, say, that its middle module is freely generated in negative degrees and its first module is freely generated in positive degrees. This means that on the level of graded $\mathbb{C}[v]$-modules, our sequence breaks up into a direct sum of copies of sequences of the type

$$
(\mathbb{C}[v])[-i] \stackrel{v^{i} ;}{\hookrightarrow}(\mathbb{C}[v])[i] \rightarrow\left(\mathbb{C}[v] /\left(v^{i}\right)\right)[i]
$$

with $i>0$. From there we see easily, that the said filtration on $\mathbb{C} \otimes_{\mathbb{C}[v]}$ $H_{\mathbb{C}^{\times}}\left(i^{!} \mathcal{I C}\right)=H\left(i^{!} \mathcal{I C}\right)$ coincides with the filtration given by its $\mathbb{Z}$-grading, and this by [KL80] is known to be given by Kazhdan-Lusztig polynomials. More precisely we get

$$
\begin{aligned}
\bar{F}^{i}\left(\mathbb{C} \otimes_{\mathbb{C}[v]} i^{!} \mathcal{I C}\right) & \cong H^{-i}(i ! \mathcal{I C}) \\
& \cong \operatorname{Der}\left(\mathcal{C}_{\bar{y}}[i], i ! \mathcal{I C}_{\bar{y}}\right) \\
& \cong \operatorname{Der}\left(i_{\bar{y} !} \mathcal{C}_{\bar{y}}[i], \mathcal{I} \mathcal{C}_{\bar{x}}\right)
\end{aligned}
$$

and this space has the dimension $h_{y, x}^{i}$ for $y, x$ the longest representatives of $\bar{y}, \bar{x}$. Thus this is the dimension of the $i$-th subquotient of the Andersen filtration on $\operatorname{Hom}_{\mathfrak{g}}\left(\Delta\left(\lambda_{\bar{y}}\right), K\left(\lambda_{\bar{x}}\right)\right)$.

\section{References}

[And97] Henning Haahr Andersen, Filtrations and tilting modules, Ann. Scient. Ecole Norm. Sup 30 (1997), 353-366.

[BB93] Alexander A. Beilinson and Joseph N. Bernstein, A proof of Jantzen conjectures, I. M. Gelfand Seminar, Adv. Soviet Math., 16, Part 1, AMS, 1993, pp. 1-50.

[BBD82] Alexander A. Beilinson, Joseph N. Bernstein, and Pierre Deligne, Faisceaux pervers, Astérisque 100 (1982), 1-172. 
[Ben91] D. J. Benson, Representations and cohomology I: Basic representation theory of finite groups and associative algebras, Cambridge Studies in Advanced Mathematics, vol. 30, Cambridge University Press, 1991.

[BG80] Joseph N. Bernstein and Sergei I. Gelfand, Tensor products of finite and infinite representations of semisimple Lie algebras, Compositio Math. 41 (1980), 245-285.

[BGG75] Joseph N. Bernstein, Israel M. Gelfand, and Sergei I. Gelfand, Differential operators on the base affine space and a study of $\mathfrak{g}$ modules, Lie groups and their Representations (I. M. Gelfand, ed.), Halsted: New York, 1975, pp. 21-64.

[BGS96] Alexander A. Beilinson, Victor Ginzburg, and Wolfgang Soergel, Koszul duality patterns in representation theory, JAMS 9 (1996), no. 2, 473-527.

[BL94] Joseph N. Bernstein and Valery Lunts, Equivariant sheaves and functors, Lecture Notes in Mathematics, vol. 1578, Springer, 1994.

[CI89] David H. Collingwood and Ron Irving, A decomposition theorem for certain self-dual modules in the category $\mathcal{O}$, Duke math. J. 58 (1989), 89-102.

[CR90] Charles W. Curtis and Irving Reiner, Methods of representation theory. Vol. I, Wiley Classics Library, John Wiley \& Sons Inc., New York, 1990, With applications to finite groups and orders, Reprint of the 1981 original, A Wiley-Interscience Publication.

[Fie06] Peter Fiebig, The combinatorics of category $\mathcal{O}$ over symmetrizable Kac-Moody algebras, Transformation Groups 11 (2006), no. 1, 2949.

[Gin91] Victor Ginzburg, Perverse sheaves and $\mathbb{C}^{*}$-actions, JAMS 4 (1991), no. 3, 483-490.

[GJ81] Ofer Gabber and Anthony Joseph, Towards the Kazhdan-Lusztig conjecture, Ann. scient. Ec. Norm. Sup. $4^{e}$ série 14 (1981), 261-302.

[KL79] David Kazhdan and George Lusztig, Representations of Coxeter groups and Hecke algebras, Inventiones 53 (1979), 191-213.

[KL80] - Schubert varieties and Poincaré duality, Proc. Symp. Pure Math. 36, AMS, 1980, pp. 185-203. 
[Soe90] Wolfgang Soergel, Kategorie $\mathcal{O}$, perverse Garben und Moduln über den Koinvarianten zur Weylgruppe, Journal of the AMS 3 (1990), 421-445.

[Soe92] - The combinatorics of Harish-Chandra bimodules, Journal für die reine und angewandte Mathematik 429 (1992), 49-74.

[Soe97a] — Charakterformeln für Kipp-Moduln über Kac-MoodyAlgebren, Representation Theory (An electronic Journal of the AMS) 1 (1997), 115-132.

[Soe97b] — Kazhdan-Lusztig-Polynome und eine Kombinatorik für Kipp-Moduln, Representation Theory (An electronic Journal of the AMS) 1 (1997), 37-68, english 83-114.

[Soe01] — Langlands' philosophy and Koszul duality, AlgebraRepresentation Theory (Roggenkamp and Stefanescu, eds.), Kluwer, 2001, Proceedings of NATO ASI 2000 in Constanta, pp. 379-414.

[Soe07] - Kazhdan-Lusztig-Polynome und unzerlegbare Bimoduln über Polynomringen, Journal de Mathématiques de l'Institut de Jussieu 6 (2007), no. 3.

[Spr84] T. A. Springer, A purity result for fixed point varieties in flag manifolds, J. Fac. Sci. Univ. Tokyo Sect. IA Math. 31 (1984), no. 2, 271-282. 